immediate income streams, often through public debt and venal offices, rather than cultivate a strong economy within the papal states. Pattenden compares papal financial strategies with those of European hereditary monarchs and concludes that customs influenced by the papacy's non-hereditary and elective nature encouraged policies that led to eventual fiscal collapse.

As befits such a complex and multifaceted thesis, this study's argument is very carefully positioned in relation to recent research. Anyone looking for a primer on the current historiography of the papacy would do well to read this volume. Not only does Pattenden explore important ideas about the papacy, but he pieces them together in order to show how they work cooperatively and reveal an institution that is more often examined one pontificate at a time than in the round. By meditating on the decision-making process that surrounded conclave, and on continued Italian support for the papacy more generally, Pattenden mobilizes research to show how current scholarly discussions are related to, and deeply impacted by, the expectation of a future election and political change. This is the great value of his book: by synthesizing decades of research and identifying the papacy's core constitutional distinction, Pattenden has illustrated how symptomatic practices grew out of, or were encouraged by, the institution's elective and non-hereditary character.

In sum, Miles Pattenden's study of the papacy builds an interesting new world out of a more holistic interpretation of the current scholarship. This is no small task, but he does it with patience and precision, and to the benefit of the larger field.

JENNIFER MARA DESILVA

Ball State University

\title{
Philadelphe, Eusèbe.
}

\section{Le Reveille-matin des François, et de leurs voisins.}

Édition de Jean-Raymond Fanlo, Marino Lambiase et Paul-Alexis Mallet. Textes de la Renaissance, 203. Paris : Classiques Garnier, 2016. 561 p. ISBN 978-2-8124-6074-6 (broché) $87 €$.

En 1576 est publiée une défense de la monarchie et du défunt Charles IX intitulée Le Vray Resveille-matin des Calvinistes et Publicains François : où est 
amplement discouru de l'auctorité des Princes, et du devoir des sujets envers iceux. Son auteur est Arnaud Sorbin; son objectif est d'invalider les prétentions d'auteurs huguenots comme Théodore de Bèze ou François Hotman. Ce faisant, l'ouvrage se concentre particulièrement sur le livre dont il détourne le titre : Le Reveille-matin des François, et de leurs voisins. Sorbin dénonce l'« impudente malice » de l'auteur qui accable le roi de "libelles diffammatoires tels que sont Alithie, Resveille-matin, [...] et autres tels fatras [...]» (511). Le Reveille-matin des François, publié entre 1573 et 1574, fit en effet l'objet de beaucoup de débats.

Jean-Raymond Fanlo, Marino Lambiase et Paul-Alexis Mallet viennent de publier cet ouvrage dans la collection « Textes de la Renaissance » aux éditions Classiques Garnier. Cette édition critique du Reveille-matin des François est la plus récente à ce jour. En France, il ne semble y en avoir eu, au XX siècle, qu'une seule autre, en 1977, aux Éditions d'histoire sociale (EDHIS). Le texte est publié en son intégralité et doté d'un imposant appareil paratextuel : l'introduction, les annexes, les références bibliographiques et les index occupent plus d'un tiers de l'ouvrage. L'introduction présente les textes dans leur contexte historique, puis commente leurs thématiques politiques et religieuses pour se concentrer ensuite sur " le problème de l'auteur " (nous ne disposons pour ce texte que d'un pseudonyme, celui d'Eusèbe Philadelphe, ou « le pieux qui aime son frère » (141). Les éditeurs font ensuite état des différentes éditions latines et françaises de l'ouvrage pour conclure avec la réception globale du Reveille-matin et les protocoles d'édition propres à cette version. En annexe, ils éditent et traduisent des pièces latines absentes des versions françaises et présentent les traductions néerlandaise et allemande ainsi que la critique d'Arnaud Sorbin. Finalement, suite aux références bibliographiques divisées entre " Ouvrages anciens » et "Références critiques ", trois index recensent 1) les noms propres, 2) les titres des ouvrages anonymes antérieurs à 1800 mentionnés par Le Reveille-matin, et 3) les références et citations bibliques.

Le premier dialogue, de 1573, initialement intitulé Dialogue auquel sont traitées plusieurs choses avenues aux Lutheriens et Huguenots de la France, certains poincts et advis nécessaires d'estre sceuz et suyvis, est d'abord paru en latin, puis en français (11). Au moment où le second dialogue est publié, en 1574, l'ouvrage prend le titre de Reveille-matin des François, et de leurs voisins : le premier dialogue se voit enrichi de nouvelles pièces (40) : une lettre de l'imprimeur aux Français et aux nations voisines, une dédicace à Élisabeth d'Angleterre, une épître aux Polonais, un dialogue sur l'effigie de la paix, une 
lettre écrite au duc de Guise et deux séries de vers : l'une destinée au chasseur déloyal - une anagramme de Charles de Valois (162) - , l'autre aux « vrais gentilshommes français ».

Philalithie (l'ami de la vérité), ayant fui la France, accompagné de l'Historiographe et du Politologue, deux Français catholiques, retrouve Alithie (la Vérité). Ensemble, l'Historiographe et le Politologue racontent les événements tragiques des guerres de religion, se disant neutres et objectifs ; ils relatent les conflits à la Vérité, qui demande : "Historiographe mon amy, sans y adjouster du tien, ny te montrer passionné pour l'un ou l'autre party : dy-nous simplement le faict » (168). Le discours de l'Historiographe débute et témoigne des persécutions perpétrées à l'endroit des huguenots au cours des différentes guerres de religion, pour conclure sur le massacre de la SaintBarthélemy. La tuerie du 24 août est narrée en détail : de l'attentat manqué de l'amiral de Coligny au massacre lui-même, et aux différents revirements royaux de Charles IX qui, d'abord, accuse les Guise pour ensuite clamer avoir pris connaissance d'un complot contre lui. Le Reveille-matin des François a été particulièrement important dans l'historiographie de la Saint-Barthélemy, développant accusations et légendes tenaces. Un passage en particulier a marqué les esprits jusqu'à aujourd'hui :

[...] et ne tarda guerres qu'ils [des seigneurs et gentilshommes huguenots] virent sur la rivière, et venir droict à eux [...] jusqu'à deux cens soldats armez de la garde du Roy, crians, Tue, tue : et leurs tirans harquebousades à la veue du Roy, qui estoit aux fenestres de sa chambre, et pouvoit estre alors environ sept heures du Dimanche matin. Encore m'a-on dict. que le Roy prenant une harquebouse de chasse entre ses mains, en reniant Dieu, dit : Tirons, mort-Dieu, ils s'enfuyent. (216)

Ce détail a contribué à la création de la légende accusant Charles IX d'avoir tiré à l'arquebuse sur des corps passant dans la Seine.

Un nouveau personnage entre alors en jeu : l'Église, qui interrompt le récit et prie pour les huguenots. Les personnages usent ensuite des vers de la Franciade de Ronsard pour critiquer Charles IX et s'attaquent à la "roynemere ", Catherine de Médicis. Finalement, Daniel, le jugement de Dieu, condamne les dirigeants français et énonce quarante articles permettant de réguler la vie des huguenots en France. L'Historiographe et le Politologue 
se convertissent, rejoignent l'Église huguenote et partent témoigner, l'un à l'étranger des injustices et massacres français, l'autre en France des quarante articles de Daniel.

Le deuxième Dialogue voit l'Historiographe et le Politologue se retrouver dans une auberge. Ils se racontent leurs périples. L'Historiographe est parti dans le Saint Empire Romain Germanique, mais surtout en Angleterre, où il a vu la reine Elisabeth menacée par Marie Stuart et les catholiques. S'ensuit un débat sur la légitimé pour un souverain d'en juger un autre. L'Historiographe continue, relatant son voyage en Suisse, qui va permettre de discuter du droit « d'occire le Tyran » (367). Le Politologue va, quant à lui, décrire l'état de la France, perdue entre les débauches royales et la déchéance morale, ainsi que narrer le siège de la Rochelle et l'arrivée des Polonais venant chercher leur nouveau souverain, le duc d'Anjou. Il achève son récit par une réflexion sur le sort du peuple « qui ayant le choix, ou d'estre serf, ou d'estre libre, [...] veut vivre sous l'iniquité, sous l'oppression [...] au seul plaisir de ce Tyran » (452), et sur celui des courtisans « contraints d'estre nuict et jour après à songer pour plaire au tyran, et se rompre, se tuer, et travailler pour inventer nouveaux moyens de trahir, de tuer, de paillarder [...]» (446). Faisant écho au Contr'un d'Étienne de la Boétie, il exhorte les Français : «mais seulement ne le soustenez plus [votre roi], et vous le verrez comme un grand Colosse, à qui on a desrobé la base, de son poix, de soy-mesme fondre en bas et se rompre »(455). Le Dialogue s'achève sur l'attente d'un messager, Spoudæe (« diligent », en grec).

Le Reveille-matin des François, et de leurs voisins est donc un ouvrage à multiples facettes. Il mêle les trois genres de la rhétorique : juridique, en relatant les événements des guerres de religion et en condamnant les persécutions des catholiques contre les huguenots, politique, en incitant le peuple français à ne plus appuyer leur roi, et épidictique, dans un discours de blâme, en s'attaquant directement à Charles IX et Catherine de Médicis. Cette pluralité fait sa complexité : c'est que Le Reveille-matin permet de saisir, dans toutes ses nuances, les divers aspects de la réaction huguenote au massacre du 24 août 1572. Il s'agit donc d'une excellente édition critique d'un texte longuement attendu pour tous ceux qui s'intéressent de près ou de loin aux répercussions de cet événement tragique de l'histoire de France. 